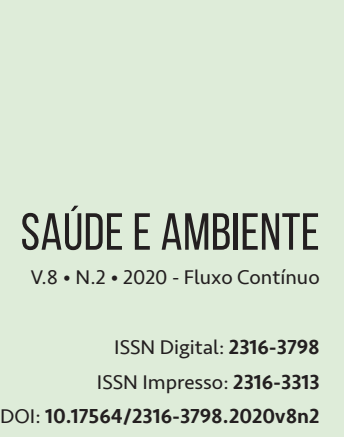

DOI: $10.17564 / 2316-3798.2020 v 8 n 2$

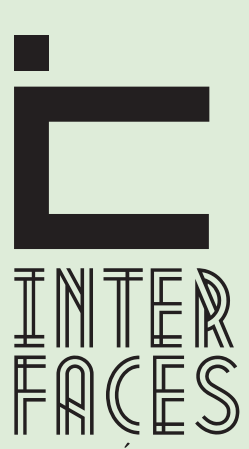

CIENTÍFICAS

\section{ANÁLISE DO PERFIL TEMÁTICO DE MONOGRAFIAS DO CURSO DE LICENCIATURA EM CIÊNCIAS BIOLÓGICAS DA UNIVERSIDADE FEDERAL DE SERGIPE [2013-2019]}

\begin{abstract}
ANALYSIS OF THE THEMATIC PROFILE OF MONOGRAPHS OF THE LICENSING COURSE IN BIOLOGICAL SCIENCES OF THE FEDERAL UNIVERSITY OF SERGIPE (2013-2019)
\end{abstract}

ANÁLISIS DEL PERFIL TEMÁTICO DE MONOGRAFÍAS DEL CURSO DE LICENCIA EN CIENCIAS BIOLÓGICAS DE LA UNIVERSIDAD FEDERAL DE SERGIPE (2013-2019]

Josean Santos Nascimento ${ }^{1}$ Ester Fraga Vilas Boas Carvalho do Nascimento ${ }^{2}$

\section{RESUMO}

A pesquisa científica é um dos pilares indubitavelmente essenciais para a formação de professores no século XXI. Com isso, o objetivo deste trabalho foi fazer a análise do perfil temático dos Trabalhos de Conclusão de Curso em formato de monografia do curso de Ciências Biológicas-Licenciatura da Universidade Federal de Sergipe (UFS). Foi realizada a busca no Repositório Institucional da UFS em que foram analisadas e lidas 81 monografias publicadas entre os anos de 2013 a 2019. Posteriormente, foi feita a quantificação das monografias por ano e suas classificações com base nas temáticas abordadas das áreas de conhecimento da Biologia, produzindo, assim, dados que foram apreciados estatisticamente pela planilha Microsoft Excel. Observou-se que $28 \%$ das monografias tratam da temática Biologia Geral, $23 \%$ das monografias da temática Ecologia, 11\% da Zoologia, 9\% de Botânica, $5 \%$ de Microbiologia, $5 \%$ de Genética, 4\% de Educação Sexual, 4\% de Paleontologia, 3\% de Evolução, 3\% de Parasitologia, 2\% de Citologia, $1 \%$ de Bioquímica, Fisiologia e Imunologia, respectivamente. As monografias desenvolvidas no Curso de Licenciatura em Ciências Biológicas da UFS têm representado importante contribuição para a pesquisa educacional, sobretudo no que se referem às questões ambientais. Todavia, algumas temáticas imprescindíveis como a Educação Sexual devem ser mais trabalhadas.

\section{PALAVRAS-CHAVE}

Ciências Biológicas. Formação de Professores. Trabalho de Conclusão de Curso. 


\section{ABSTRACT}

Scientific research is one of the undoubtedly essential pillars for teacher training in the 21st century. With that, the objective of this work was to analyze the thematic profile of the Course Conclusion Papers in monograph format of the Biological Sciences course at the Federal University of Sergipe. A search was made in the Institutional Repository of UFS in which 81 monographs published between the years 2013 to 2019 were analyzed and read. Subsequently, the monographs were quantified by year and their classifications based on the topics covered in the areas of knowledge of Biology, thus producing data that was statistically appreciated by the Microsoft Excel spreadsheet. It was observed that $28 \%$ of the monographs deal with the theme General Biology, $23 \%$ of the monographs with the theme Ecology, $11 \%$ of Zoology, $9 \%$ of Botany, $5 \%$ of Microbiology, $5 \%$ of Genetics, $4 \%$ of Sexual Education, $4 \%$ Paleontology, 3\% Evolution, 3\% Parasitology, 2\% Cytology, 1\% Biochemistry, Physiology and Immunology, respectively. The monographs developed in the Biological Sciences Degree Course at UFS have represented an important contribution to educational research, especially with regard to environmental issues. However, some essential themes, such as Sex Education, should be further worked on.

\section{KEYWORDS}

Biological Sciences, Teacher Training, Course Completion Work.

\section{RESUMEN}

La investigación científica es sin duda uno de los pilares fundamentales para la formación del profesorado en el siglo XXI. Con ello, el objetivo de este trabajo fue analizar el perfil temático de los Trabajos de Conclusión del Curso en formato monográfico del curso de Ciencias Biológicas - Licenciatura de la Universidad Federal de Sergipe. Se realizó una búsqueda en el Repositorio Institucional de la UFS en la que se analizaron y leyeron 81 monografías publicadas entre los años 2013 a 2019. Posteriormente, se cuantificaron las monografías por año y sus clasificaciones en base a los temas tratados en las áreas de conocimiento de Biología, produciendo así datos que fueron estadísticamente apreciados por la hoja de cálculo de Microsoft Excel. Se observó que el $28 \%$ de las monografías tratan el tema Biología General, el 23\% de las monografías con el tema Ecología, el 11\% de Zoología, el 9\% de Botánica, el 5\% de Microbiología, el 5\% de Genética, el 4\% de Educación Sexual, el 4\% Paleontología, 3\% Evolución, 3\% Parasitología, 2\% Citología, 1\% Bioquímica, Fisiología e Inmunología, respectivamente. Las monografías desarrolladas en la Licenciatura en Ciencias Biológicas de la UFS han representado un importante aporte a la investigación educativa, especialmente en materia ambiental. Sin embargo, es necesario seguir trabajando en algunos temas fundamentales, como la educación sexual. 


\section{PALABRAS CLAVE}

Ciencias biológicas, formación del profesorado, trabajo de finalización del curso.

\section{INTRODUCÇÃO}

Desde os mestres da civilização grega, as Missões Jesuíticas no Brasil, no século XVI, de cunho catequizador e educativo e, os professores régios pagos com o erário público, instituídos pela Reforma Pombalina em Portugal em suas colônias entre os séculos XVI e XVIII, são constantes as transformações da função professoral ao longo do tempo (BORTOLINI; NUNES, 2018). Na contemporaneidade isto não é diferente, sendo a pesquisa científica um dos pilares indubitavelmente essenciais para a formação de professores no século XXI.

0 mundo atual compelido de imensuráveis inovações exige que a função docente ultrapasse o ideário tradicionalista do mero repasse de conteúdos, e que o professor como mediador e facilitador do conhecimento hoje difundido nos mais diversos meios digitais, instigue nos educandos o desenvolvimento do senso crítico, reflexivo e pesquisador para que, desta maneira, tenha-se indivíduos protagonistas da construção do conhecimento e atores ativos da realidade social na qual estão inseridos.

A pesquisa científica enriquece sobremaneira o cotidiano da sala de aula dos docentes, expandindo os saberes adquiridos na formação inicial e facilitando o processo ensino-aprendizagem. Freire (1996, p. 16), ressalta a relação da pesquisa no âmbito do ensino. Para o referido teórico,

Não há ensino sem pesquisa e pesquisa sem ensino. Esses que-fazeres se encontram um no corpo do outro. Enquanto ensino continuo buscando, reprocurando. Ensino porque busco, porque indaguei, porque indago e me indago. Pesquiso para constatar, constatando, intervenho, intervindo educo e me educo. Pesquiso para conhecer o que ainda não conheço e comunicar ou anunciar a novidade.

A pesquisa tem importância no decorrer da graduação e no exercício docente, pois estes profissionais contribuem para a educação, realizando questionamentos de problemáticas educacionais ainda não pesquisadas, bem como, produzindo inovações metodológicas na Educação Básica (SILVA; SANTOS, 2017). Entretanto, para a consolidação de ações de pesquisa científica no fazer professoral, é imprescindível que durante sua formação inicial os futuros educadores sejam inseridos no campo da pesquisa. A Lei de Diretrizes e Bases da Educação Nacional (LDBEN - Lei n. 9394/96 que estabelece as diretrizes e bases da educação nacional) em seu Art. 43, enfatiza que algumas das finalidades da Educação Superior no Brasil são as de

I - estimular a criação cultural e o desenvolvimento do espírito científico e do pensamento reflexivo; II - formar diplomados nas diferentes áreas de conhecimento, aptos para a inserção em setores profissionais e para a participação no desenvolvimento da sociedade 
brasileira, e colaborar na sua formação contínua; III - incentivar o trabalho de pesquisa e investigação científica, visando o desenvolvimento da ciência e da tecnologia e da criação e difusão da cultura, e, desse modo, desenvolver o entendimento do homem e do meio em que vive; IV - promover a divulgação de conhecimentos culturais, científicos e técnicos que constituem patrimônio da humanidade e comunicar o saber através do ensino, de publicações ou de outras formas de comunicação [...]. (BRASIL, 1996, p. 32).

Neste contexto, destaca-se o Trabalho de Conclusão de Curso (TCC) como um dos instrumentos que podem ser primordiais para a realização da pesquisa científica durante a graduação. Além de representarem um dos principais meios para que os acadêmicos sejam inseridos no âmbito da pesquisa, os TCC podem definir o senso crítico e investigativo em sua profissionalização. Entretanto, é importante ressaltar que a depender das instruções normativas específicas das Instituições de Ensino Superior (IES) o TCC pode não se caracterizar como pesquisa científica, algumas IES, por exemplo, utilizam o relatório de Estágio Supervisionado como Trabalho de Conclusão de Curso.

Mesmo com todas as dificuldades e questões emocionais simultâneas a elaboração do TCC, este representa um momento de formação profissional excelente para a qualificação e posterior inserção do acadêmico num mercado de trabalho cada vez mais competitivo, pois evidencia a fundamentação teórica e prática do futuro profissional (TRINDADE et al., 2018).

No que concerne aos Cursos de Licenciatura em Ciências Biológicas, as Diretrizes Curriculares Nacionais estabelecem que, entre outras competências, o profissional desta área deve "atuar em pesquisa básica e aplicada nas diferentes áreas das Ciências Biológicas, comprometendo-se com a divulgação dos resultados das pesquisas em veículos adequados para ampliar a difusão e ampliação do conhecimento” (BRASIL, 2001, p. 3).

Assim sendo, os TCC, representando pré-requisito parcial para a obtenção do título acadêmico almejado, criam a possibilidade dos futuros professores aprimorar o desenvolvimento de sua práxis pedagógica e o conhecimento do contexto escolar em suas múltiplas faces. Com isso, a escolha da temática a ser investigada nos TCC é imprescindível para a divulgação científica na sociedade e para a consolidação da epistemologia adquirida na graduação pelos discentes.

A temática desenvolvida pelos discentes em seus TCC apesar de muitas vezes refletirem sua proximidade com o assunto, nem sempre corroboram a continuidade de sua elaboração, pois a possibilidade de efetivação do estudo e a base teórica sobre a temática podem ser relegadas (YAMANARI; MORAES, 2013). Desse modo, a avaliação crítica e racional da temática a ser escolhida para o TCC é um fator preponderante para o posterior desenvolvimento do mesmo.

O Curso de Licenciatura em Ciências Biológicas da Universidade Federal de Sergipe (UFS) é o único oferecido por uma Instituição Pública de Ensino Superior no Estado de Sergipe. A história do Curso de Ciências Biológicas da UFS se inicia com a criação do chamado Instituto de Biologia, em 12 de março de 1969, entretanto, o curso iniciou suas atividades no ano de 1972 e foi reconhecido oficialmente apenas no ano de 1977, por meio do Decreto nº 79.947 (CARMO, 2011).

Este Curso é ofertado atualmente, de forma presencial no Campus São Cristóvão (Cidade Universitária Prof. José Aloísio de Campos) e no Campus Prof. Alberto Carvalho, em Itabaiana, além de ser ofertado à distância em polos localizados em algumas cidades do Estado. 
Desta forma, o presente trabalho objetivou fazer a análise do perfil temático dos Trabalhos de Conclusão de Curso em formato de monografia do curso de Ciências Biológicas-Licenciatura da Universidade Federal de Sergipe. Este objeto de pesquisa se justifica pela necessidade do fornecimento de dados essenciais acerca dos processos educativos que permeiam o futuro professor de Ciências ou Biologia em fase de conclusão de curso para a realização de pesquisas subsequentes, bem como por fornecer subsídios para a comunidade acadêmica compreender a atual realidade do ensino de Ciências e de Biologia em nosso país nos Trabalhos de Conclusão de Curso.

\section{PROCEDIMENTOS METODOLÓGICOS}

Esta pesquisa preza pela abordagem quali-quantitativa de cunho exploratório e descritivo, caracterizada quanto aos procedimentos utilizados como análise documental. As pesquisas são categorizadas em quali-quantitativas quando apresentam tanto mensuração dos dados coletados como o estudo dos aspectos qualitativos. De acordo com Gil (2002, p. 41-42), pesquisas de cunho exploratório "têm como objetivo principal o aprimoramento de ideias ou a descoberta de intuições" e as pesquisas descritivas "têm como objetivo primordial a descrição das características de determinada população ou fenômeno". Pesquisas que se utilizam de análise documental são elaboradas a partir da analise de materiais que ainda não haviam recebido um tratamento analítico (RODRIGUES et al., 2011).

A Universidade Federal de Sergipe teve sua criação instituída em 28 de fevereiro de 1967, pelo Decreto-Lei no 269, e efetivada em 1968, com a aprovação do Conselho Federal de Educação de seu primeiro estatuto (ARAÚJO, 2008). O Departamento de Biologia (DBI) da instituição contempla os Cursos de Licenciatura e de Bacharelado em Ciências Biológicas. Contudo, por meio do Repositório Institucional da UFS (RI/UFS), existente em um canal digital, o presente trabalho se deteve a pesquisar monografias publicadas que fossem referentes ao Curso de Licenciatura em Ciências Biológicas.

O Repositório Institucional da Universidade Federal de Sergipe é um meio utilizado para a difusão do conhecimento acadêmico e científico produzido na instituição de ensino. No mesmo são disponibilizados dissertações, teses, monografias e outros trabalhos acadêmicos elaborados na universidade. Portanto, por seu fácil acesso este repositório representa um eminente mecanismo para a disseminação do conhecimento científico nas mais diversas esferas sociais.

Inicialmente realizou-se a busca no Repositório Institucional utilizando o descritor "Ensino Biologia” e o marco temporal 2013 a 2019. Desse modo, foram encontradas 163 monografias, todavia, somente 81 destas monografias eram concernentes ao Curso de Licenciatura em Ciências Biológicas. De acordo com Prodanov e Freitas (2013, p. 155), “o trabalho monográfico caracteriza-se mais pela unicidade e delimitação do tema e pela profundidade do tratamento do que por sua eventual extensão, generalidade ou seu valor didático”.

Posteriormente, foi feita a quantificação das monografias por ano e suas classificações com base nas temáticas de ensino das áreas de conhecimento relacionadas a Ciências Biológicas postuladas pelo CNPq, sendo que foi adicionada para a classificação a área temática Educação Sexual. Bem 
como, foi analisada a ênfase dada às monografias a temas como Educação Ambiental, Educação Inclusiva e Formação de professores.

\section{RESULTADOS E DISCUSSÃO}

Os resultados demonstram um número não linear de publicações de TCC do curso de Licenciatura em Ciências Biológicas no RI/UFS. Houve uma quantidade maior de publicações no ano de 2018 (25 monografias) e uma quantidade menor nos anos de 2013, 2014 e 2015 (uma monografia em cada ano), conforme a Figura 1.

Figura 1 - Número de TCCs publicados no RI/UFS entre os anos de 2013 a 2019

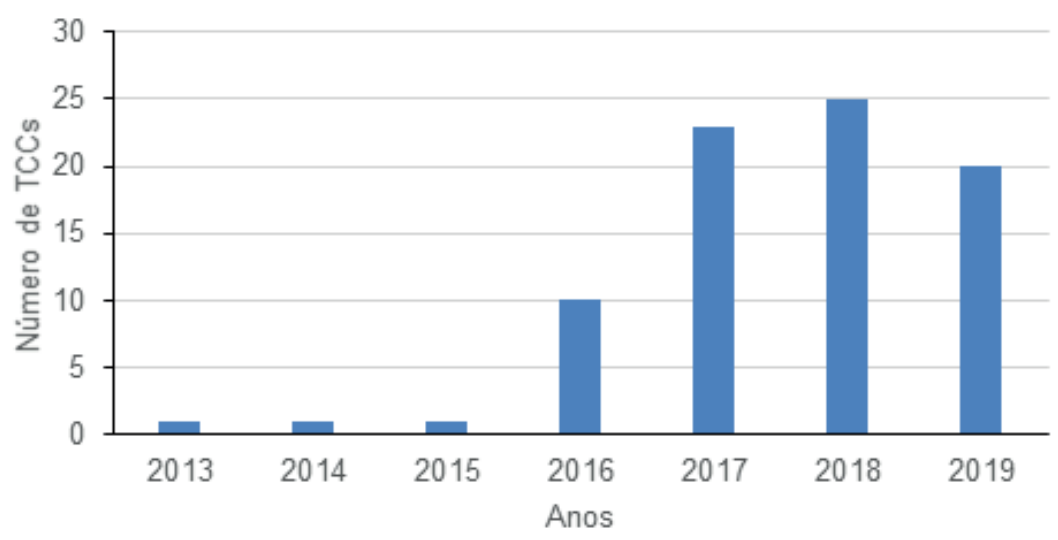

Fonte: RI/UFS (2019).

Nos referidos anos em que apenas um TCC foi publicado, possivelmente houve a ocorrência de incongruências entre o número de TCC apresentados e o encaminhamento de tais trabalhos com a assinatura do termo de autorização do autor para que as Bibliotecas dos Campus da UFS, setor administrativo que é responsável pelas publicações no repositório, procedessem ao processo de publicação destes TCC.

Portanto, o número de TCC no Repositório da UFS reflete o número de formandos que encaminham seus trabalhos para a biblioteca do seu campus realizar a publicação no repositório institucional.

Realizou-se o cálculo da média aritmética simples de TCC de Licenciatura em Ciências Biológicas publicados no RI/UFS por ano, obtendo-se a média de 11,6 TCC.

Um dos problemas corriqueiros nas Universidades é a ultrapassagem do tempo médio de integralização dos cursos pelos alunos. Sendo assim, a produção de TCC acaba sendo relegada para períodos anteriores ou posteriores ao recomendado na grade curricular. A UFS fornece dados pertinentes acerca desta problemática. Um desses dados é a Taxa de Sucesso dos Cursos que é "um indicador anual que de- 
monstra a porcentagem de diplomados, em um referido ano, em relação ao total de ingressantes retroativos, considerando o tempo mínimo de integralização de cada curso" (OLIVEIRA; SANTOS, 2019, p. 47).

Nos anos de publicações de TCC analisados neste trabalho (com exceção do ano de 2019, pois o Anuário Estatístico referente a este ano não foi publicado até a conclusão deste trabalho e os TCC apresentados neste ano refletem o ano anterior em decorrência do atraso do calendário acadêmico da instituição de ensino), o curso de Licenciatura em Ciências Biológicas na modalidade presencial obteve as Taxas de Sucesso apresentadas no Quadro 1.

Quadro 1 - Taxa de Sucesso do Curso de Ciências Biológicas - Licenciatura - modalidade presencial da UFS entre os anos de 2013 e 2018

\begin{tabular}{|c|c|c|c|}
\hline Curso & Turno & Campus & Ano - Taxa de Sucesso \\
\hline \multirow{18}{*}{$\begin{array}{c}\text { Ciências Biológicas } \\
\text { Licenciatura }\end{array}$} & \multirow{6}{*}{ Diurno } & \multirow{6}{*}{ São Cristovão } & $2013-48,78 \%$ \\
\hline & & & $2014-58,33 \%$ \\
\hline & & & $2015-16,67 \%$ \\
\hline & & & $2016-39,00 \%$ \\
\hline & & & 2017 - 58,54\% \\
\hline & & & $2018-46,34 \%$ \\
\hline & \multirow{6}{*}{ Noturno } & \multirow{6}{*}{ São Cristovão } & $2013-65,45 \%$ \\
\hline & & & $2014-30,00 \%$ \\
\hline & & & $2015-35,19 \%$ \\
\hline & & & $2016-31,00 \%$ \\
\hline & & & $2017-40,00 \%$ \\
\hline & & & $2018-69,23 \%$ \\
\hline & \multirow{6}{*}{ Diurno } & \multirow{6}{*}{ Itabaiana } & $2013-49,28 \%$ \\
\hline & & & $2014-50,85 \%$ \\
\hline & & & $2015-58,49 \%$ \\
\hline & & & $2016-66,00 \%$ \\
\hline & & & $2017-67,21 \%$ \\
\hline & & & $2018-56,52 \%$ \\
\hline
\end{tabular}

Fonte: Adaptado de Anuários Estatísticos da UFS (2019).

No Brasil existem alguns estudos que abordam a análise de temáticas de Trabalhos de Conclusão de Curso. Exemplos destes trabalhos são os de Teixeira (2016), que investigou TCC de um curso de 
Licenciatura em Educação Física e, Faustino e colaboradores (2019), que trataram de temáticas de TCC de um Curso de Pedagogia. No campo da Licenciatura em Ciências Biológicas existem também alguns trabalhos que traçam o perfil temático de TCC, como o de Chagas e colaboradores (2017) e Sousa e Lemos (2018). Entretanto, não foi encontrado na literatura nenhum trabalho que abordasse a análise de temáticas de TCC do curso de Licenciatura em Ciências Biológicas da UFS, o que indica o caráter inédito do presente trabalho.

Com base nos TCC analisados, pode-se constatar que a temática da área do conhecimento mais tratada nas monografias deste estudo foi a da Biologia Geral presente em $28 \%$ dos TCC (23), seguido da área da Ecologia tratada em $23 \%$ dos TCC (19) e da Zoologia em 11\% dos TCC (9). As temáticas menos abordadas nos TCC foram Bioquímica, Fisiologia e Imunologia presentes respectivamente em $1 \%$ dos TCC (1). Outros resultados obtidos estão expostos na Figura 2.

Figura 2 - Temáticas abordadas nos TCCs do curso de Ciências Biológicas - Licenciatura - da Universidade Federal de Sergipe entre 2013 e 2019

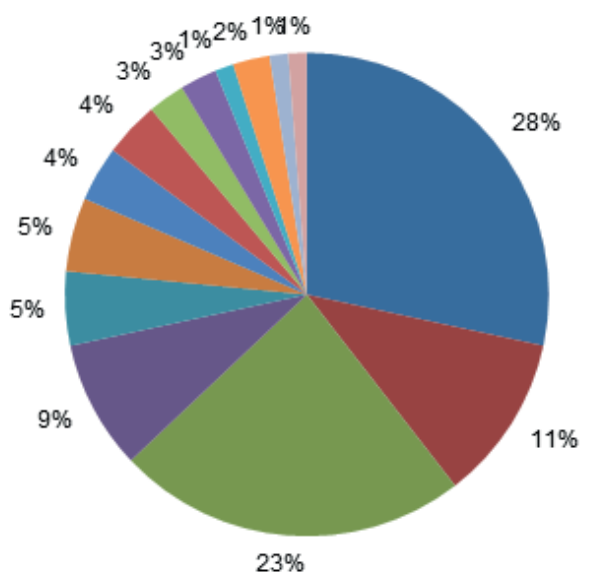



Fonte: RI/UFS, 2019.

Dos 23 TCC com perfil temático da Biologia Geral, dois apresentaram abordagem prática da formação de professores, quatro tinham ênfase na prática de Educação Ambiental e cinco em Educação Inclusiva. Os TCC de Ecologia abordavam majoritariamente os processos de ensino e práticas da Educação Ambientais (14 TCC dos 19 com a temática Ecologia) e somente dois com abordagem da Educação Inclusiva. Dos nove TCC de Zoologia, apenas dois tinham ênfase na prática de Educação Ambiental.

A concentração do Perfil Temático dos TCC nestas áreas de conhecimento das Ciências Biológicas pode ser explicada pelo fato de que tais temáticas são mais recorrentes durante a graduação, com a realização geralmente de atividades complementares e extensionistas que motivam os graduandos ao interesse e desenvolvimento de estudos mais acurados nestas áreas. 0 pouco número de TCC com abordagem na formação de professores ratifica o desinteresse corriqueiro da comunidade acadêmica 
acerca do seu próprio processo formativo e assim, aponta a essencialidade do graduando refletir sobre a importância de uma formação qualificada.

Um dos TCC sobre Paleontologia propôs a aplicação de um ambiente virtual de aprendizagem por meio do desenvolvimento de um blog com conteúdos e atividades referentes à Paleontologia. Outro TCC, este da temática Zoologia, aplicou Modelos 3D de museus de História Natural para a contribuição do aumento da motivação e aprendizagem da Zoologia em sala de aula. Desse modo, constata-se a inovação metodológica para educação básica que podem surgir a partir de pesquisas realizadas em Trabalho de Conclusão de Curso.

Pedrotti e Strohschoen (2015), fazendo análise das temáticas de TCC dos cursos de licenciatura e bacharelado em Ciências Biológicas, apresentados entre 2005 e 2015 em um Centro Universitário do Rio Grande do Sul, obtiveram o resultado de $34 \%$ dos TCC discutindo temas de Ecologia. Tal resultado destoa do observado na presente pesquisa em que apenas $23 \%$ dos TCC (19) tratavam deste tema.

Chagas e colaboradores (2017), explorando os temas de 136 TCC produzidos entre os anos de 2010 e 2015, por acadêmicos do curso de Licenciatura em Ciências Biológicas da Universidade do Estado do Amazonas, observaram que as áreas de Ecologia, Zoologia e Botânica são desenvolvidas cada uma em 18 monografias. Todavia, os autores ressaltam que estas temáticas se restringem às especificidades da Biologia e não concretamente ao campo educacional.

Contrariamente ao identificado nas monografias do presente estudo, pois todas as monografias estavam concatenadas ao âmbito educacional, mesmo debatendo inúmeros assuntos inerentes ao conhecimento biológico. As 81 monografias analisadas traziam abordagem das nuances que cercam o processo educativo escolar, como avaliação de temáticas de Biologia em livros didáticos, desenvolvimento de ferramentas pedagógicas para determinados objetos de conhecimento da Biologia, avaliação do processo formativo do professor de Ciências ou Biologia, práticas educativas de sensibilização ambiental etc.

Sousa e Lemos (2018), avaliando o perfil temático de TCC do curso de Licenciatura em Ciências Biológicas da Universidade Federal do Piauí elaborados entre 2010 e 2016, verificaram que a maioria trata de temas educacionais, corroborando o resultado do corrente estudo em que todas as monografias discutem o meio educativo.

\section{CONSIDERAÇÕES FINAIS}

O ímpeto investigativo e pesquisador do indivíduo durante a graduação e na prática profissional enriquecem grandemente sua formação tanto como ser humano quanto como profissional. No fazer professoral em que o docente cotidianamente se depara com situações a serem pesquisadas, este enriquecimento contribui também para a elevação da qualidade do ensino e do processo de ensino aprendizagem.

Com base nos resultados apresentados, conclui-se que abrangendo 14 áreas da Biologia, as monografias desenvolvidas no Curso de Licenciatura em Ciências Biológicas da UFS, têm representado importante contribuição para a pesquisa educacional, sobretudo no que se referem às questões da 
Educação Ambiental. Todavia, algumas temáticas imprescindíveis como a Educação Sexual devem ser mais trabalhadas para uma mudança social efetiva que o conhecimento e a pesquisa científica são capazes de proporcionar, uma vez que o Estado de Sergipe figura como um dos Estados com maior frequência de gravidez na adolescência.

\section{REFERÊNCIAS}

ARAÚJO, S. G. A instalação da Universidade Federal de Sergipe: 0 ensino de graduação sob o signo da Reforma Universitária. Congresso Brasileiro de História da Educação, 5, 2008. Anais [...], Aracaju: UNIT/SBHE, 2008. Disponível em: http://www.sbhe.org.br/novo/congressos/cbhe5/ pdf/154.pdf. Acesso em: 11 maio 2020.

BRASIL. Ministério de Educação e Cultura. LDB - Lei n 9394/96, de 20 de dezembro de 1996. Estabelece as diretrizes e bases da Educação Nacional. Brasília: MEC, 1996. Disponível em: https://www2.senado. leg.br/bdsf/bitstream/handle/id/529732/lei_de_diretrizes_e_bases_1ed.pdf. Acesso em: 15 maio 2020.

BRASIL, Ministério da Educação e Cultura, Conselho Nacional de Educação, Câmara de Educação Superior. Diretrizes Curriculares para os Cursos de Ciências Biológicas. CNE. Parecer CNE/CES 1.301/2001, Diário Oficial da União de 7/12/2001, Seção 1, p. 25. Disponível em: http://portal. mec.gov.br/cne/arquivos/pdf/CES1301.pdf. Acesso em: 5 abr. 2020.

BORTOLINI, R. W.; NUNES, C. A Paideia grega: aproximações teóricas sobre o ideal de formação do homem grego. Filosofia e Educação, Campinas, v. 10, n. 1, p. 21-36, 2018. Disponível em: https:// periodicos.sbu.unicamp.br/ojs/index.php/rfe/article/view/8651997. Acesso em: 11 maio 2020. CARMO, K.A. Uma história do curso de Ciências Biológicas na Universidade Federal de Sergipe: Para quê? O quê? Para quem? Como? (1969-1983). 2011. 128 f. Dissertação (Mestrado em Educação) - Universidade Federal de Sergipe, São Cristovão, 2011. Disponível em: https://ri.ufs.br/bitstream/ riufs/4679/1/KATIA_\%20ARAUJO_CARMO.pdf. Acesso em: 11 maio 2020.

CHAGAS, J. et al. Análise das temáticas dos Trabalhos de Conclusão de Curso de Licenciatura em Ciências Biológicas na Universidade do Estado do Amazonas. In: Congresso Nacional de Pesquisa e Ensino de Ciências, 2, 2017. Anais [...], Campina Grande: Realize, 2017. Disponível em: http:// www.editorarealize.com.br/revistas/conapesc/trabalhos/TRABALHO_EV070_MD1_SA21_ ID1952_15052017114656.pdf. Acesso em: 12 maio 2020.

FAUSTINO, R. A. C. et al. A produção discente e o panorama temático dos trabalhos de conclusão de curso de Pedagogia da UEL (2008-2016). Rev. Ens. Educ. Ciên. Hum., v. 20, n. 3, p. 312-318, 2019. Disponível em: https://seer.pgsskroton.com/index.php/ensino/article/view/6982/4801. Acesso em: 13 maio 2020. 
FREIRE, P. Pedagogia da autonomia: saberes necessários à prática educativa. 25 ed. São Paulo: Paz e Terra, 1996.

GIL, A. C. Como elaborar projetos de pesquisa. 4. ed. São Paulo: Atlas, 2002.

OLIVEIRA, K. F.; SANTOS, R. F. (org.). Anuário estatístico da UFS: 2016-2018. São Cristovão: COPAC/PROPLAN, 2019. Disponível em: http://indicadores.ufs.br/uploads/page_attach/path/7127/ Anu_rio_Estat_stico_da_UFS_2016-2017-2018_final.pdf. Acesso em: 14 maio 2020.

\section{PEDROTTI, J.; STROHSCHOEN, A. A. G. Trabalhos de conclusão de curso de ciências biológicas -} temáticas principais. 2015. TCC (Bacharelado em Ciências Biológicas) - Universidade do Vale do Taquari - Univates, Lajeado, 2015. Disponível em: http://hdl.handle.net/10737/1210. Acesso em: 12 maio 2020.

PRODANOV, C. C.; FREITAS, E. C. Metodologia do trabalho científico: métodos e técnicas da pesquisa e do trabalho acadêmico. 2. ed. Novo Hamburgo: Feevale, 2013.

REPOSITÓRIO Institucional da Universidade Federal de Sergipe (RIUFS). Disponível em: https:// ri.ufs.br/. Acesso em: 18 mar. 2020.

RODRIGUES, A.J. Metodologia científica. 4. ed. Aracaju: Edunit, 2011.

SILVA, C. A.; SANTOS, W. L. A Pesquisa na prática docente: dilemas na contemporaneidade. Rev. Rios., v. 12, p. 22-34, 2017. Disponível em: https://www.fasete.edu.br/revistarios/media/revistas/2017/12/a_ pesquisa_na_pratica_docente_dilemas_na_contemporaneidade.pdf. Acesso em: 11 maio 2020.

SOUSA, L. L. L.; LEMOS, J. R. Perfil dos trabalhos de conclusão de curso (TCCs) do Curso de Licenciatura em Ciências Biológicas da Universidade Federal do Piauí - Campus Ministro Reis Velloso (Brasil). Rev. Espacios, v. 39, n. 29, p. 1-9, 2018. Disponível em: https://www. revistaespacios.com/a18v39n29/a18v39n29p04.pdf. Acesso em: 12 maio 2020.

\section{TEIXEIRA, C. G. Mapeamento dos trabalhos de conclusão de curso de licenciatura em educação}

física na UFPel. 2016. 60 f. Dissertação (Mestrado em Educação Física) - Universidade Federal de Pelotas, Pelotas, 2016. Disponível em: http://guaiaca.ufpel.edu.br/bitstream/prefix/3754/1/ Carin\%20Gomes\%20Teixeira.pdf. Acesso em: 13 maio 2020.

TRINDADE, A. P. N. T. et al. TCC: um momento obrigatório ou uma oportunidade construída? Rev. Triângulo, v. 11, n. 1, p. 225-234, 2018. Disponível em: http://seer.uftm.edu.br/revistaeletronica/ index.php/revistatriangulo/article/download/2720/pdf_1. Acesso em: 6 maio 2020. 
YAMANARI, T. T.; MORAES, D. A. F. O papel do TCC na formação de estudantes universitários. Jornada de Didática e I Seminário de Pesquisa da CEMAD, 2, Londrina, 2013. Anais [...], Londrina: Universidade Estadual de Londrina, 2013. Disponível em: http://www.uel.br/eventos/ jornadadidatica/pages/arquivos/II\%20Jornada\%20de\%20Didatica\%20e\%20I\%20Seminario\%20 de\%20Pesquisa\%20do\%20CEMAD\%20-\%20Docencia\%20na\%20educacao\%20Superior\%20 caminhos\%20para\%20uma\%20praxis\%20transformadora/O\%20PAPEL\%20D0\%20TCC\%20 NA\%20FORMACAO\%20DE\%20ESTUDANTES\%20UNIVERSITARIOS.pdf. Acesso em: 13 maio 2020. 
1 Acadêmico em Ciências Biológicas - Licenciatura da Universidade Tiradentes - UNIT/SE; Membro do Grupo de Pesquisa História das Práticas Educacionais - GPHPE/ UNIT; Bolsista de Iniciação Científica - PROBIC/UNIT. E-mail: joseansantos03@gmail.com

2 Doutora em Educação; Professora do Programa de Pós-graduação em Educação e do Curso de Ciências Biológicas da Universidade Tiradentes - UNIT/SE; Bolsista de Produtividade CNPQ nível 2. E-mail: esterfraga@gmail.com

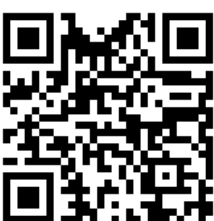

A autenticidade desse artigo pode ser conferida no site https://periodicos. set.edu.br

\section{(2) (1) (-)}

Este artigo é licenciado na modalidade acesso aberto sob a Atribuição-Compartilha Igual CC BY-SA

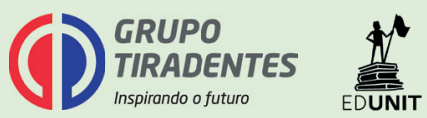

